\title{
Message From the Editors to Our Reviewers
}

José G. Merino, MD, MPhil, Olga Ciccarelli, MD, PhD, FRCP, Bradford B. Worrall, MD, MSc, Anthony A. Amato, MD, Rebecca Burch, MD, Stacey L. Clardy, MD, PhD, Peter Hedera, MD, PhD, Linda A. Hershey, MD, PhD, Barbara C. Jobst, MD, PhD, Renée A. Shellhaas, MD, MS, Elizabeth Silbermann, MD, Roy E. Strowd, III., MD, and Rawan Tarawneh, MD

Neurology ${ }^{\circledR}$ 2022;98:3-11. doi:10.1212/WNL.0000000000013044

The editors and editorial team of Neurolog $y^{\circledR}$ express our thanks to the dedicated peer reviewers who have contributed time and expertise over the past 6 months. Decisions about which papers to publish are informed by their evaluation of the novelty, accuracy, interpretation, and implications of research questions, methods, and results. The high quality and success of this journal owe much to their continuing dedication.

In the 6-month interval from April 1 through September 30, 2021, Neurology received 2,946 new and 650 revised manuscripts, and the reviewers listed in this article completed 5,379 reviews. For comparison, the figures for the same period in 2019 were 2,718 new manuscripts, 597 revised manuscripts, and 4,454 reviews. Nevertheless, the time that reviewers took to complete their work decreased in 2021, down to 20.5 days compared to 22 days in 2020.

This year we are also instituting an acknowledgement of contributors to the digital podcasts. The Neurology ${ }^{\circledR}$ podcast, one of the first podcasts in medical education, was founded in 2007 by Ted Burns, MD, and has grown to reach an average of 15,865 weekly downloads in 2021. In 2019, the Neurology Minute ${ }^{\circledR}$ was launched in response to the evolving nature of broadcast audio, to provide clinically relevant neurology news in 1-2 minutes of concise audio briefing. Thanks to the contributions of Editor Stacey Clardy, MD, PhD, FAAN, Deputy Editor Jeff Ratliff, MD, and the interviewer producers of the Podcast team, our podcasts provide unique insights that affect clinical practice and patient care in an engaging and convenient format.

If you would like to contribute more reviews, or if you have never reviewed for the journal but are interested in doing so, please send an email to journal@neurology.org. For guidance in reviewing, see the Information for Reviewers (IFR) link at n.neurology.org/submit/submitreview. Click on this link for information on expectations of reviewers regarding confidentiality, timeliness, and reviewer conflicts of interest. The IFR also provides instructions for formatting the comments to editors and authors to make communication with authors most effective.

The reviewers listed below with 1 asterisk have reviewed 5 or more papers between April 1 and September 30, 2021. Two asterisks indicate that the reviewer has reviewed 10 or more manuscripts during this period. This list includes those reviewers who returned a review or reviews of initial submissions (re-reviews of the same manuscript are not included).

$\begin{array}{lll}\text { Ashley Aaroe } & \text { Sarah Ackley } & \text { Federica Agosta* } \\ \text { Dag Aarsland } & \text { Gyula Acsadi } & \text { Sonal Agrawal } \\ \text { Hesham Abboud } & \text { Harold P. Adams } & \text { Rebekah Ahmed } \\ \text { Hisham Abdelmotilib } & \text { David D. Adams } & \text { Nayeon Ahn } \\ \text { Wilson F. Abdo } & \text { Babatunde Adeyemo } & \text { Umer Akbar } \\ \text { Taylor J. Abel } & \text { Malik Adil } & \text { Gülden Akdal } \\ \text { Erin L. Abner } & \text { Jennifer Adrissi } & \text { Rizwan S. Akhtar } \\ \text { Bassel W. } & \text { Busranur Agac* } & \text { Steven M. Albert* } \\ \text { Abou-Khalil } & \text { Nitin Agarwal } & \text { Roger L. Albin* }\end{array}$

\author{
Megan Christine \\ Alcauskas* \\ Eva C. Alden \\ Chad M. Aldridge \\ Andrei V. Alexandrov \\ Alicia Algeciras- \\ Schimnich \\ Farwa Ali* \\ Ayham Alkhachroum
}

Louise M. Allan Jeffrey A. Allen* Talal Al-Mayhani Håkan Almqvist Michael L. Alosco Saud Alsahli Qasem N. Alshaer Rustam Al-Shahi Salman 


\begin{tabular}{|c|c|c|}
\hline Claudia Altamura & Antoaneta Balabanov & Frank Besag \\
\hline Enrique Alvarez & Robert W. Baloh & Jessica M. Besbris \\
\hline Maria Pia Amato & Miriam Melissa & Lauren A. Beslow* \\
\hline Faisal Mohammad & Baltuano & David Q. Beversdorf \\
\hline Mohammad Amin & Songhurst* & May A. Beydoun \\
\hline Catherine M. Amlie- & Veera Venkata & Poonam Bhatia \\
\hline Lefond & Ratnam Bandaru & Ritwik Bhatia* \\
\hline Pria Anand & Oliver Bandmann & Rohini Bhole \\
\hline Beau M. Ances* & Gargi Banerjee & Christian G. Bien \\
\hline Oluf Andersen & Tallie Z. Baram & Geert Jan Biessels \\
\hline Craig Anderson & Michela Barichella & Alessandro Biffi \\
\hline Christopher D. & Frederik Barkhof** & Philippe Bijlenga \\
\hline Anderson & Carolina Barnett & Murat Bilgel \\
\hline Danielle M. Andrade & Christian Barro & Michel M. Billiard \\
\hline Corrado I. Angelini* & Fabrice Bartolomei & Malcolm Binns \\
\hline David Joseph & Brandon R. Barton & Gerard N. Bischof* \\
\hline Anschel & Joshua I. Barzilay & Alexander R. Bisdorff \\
\hline Kaarin J. Anstey & Rifaat M. Bashir & Andrew Bivard \\
\hline Robert M. Anthenelli & Martin Bauer & Craig Blackstone \\
\hline Angelo Antonini & Stephanie Baulac & Leah J. Blank \\
\hline Yaacov Anziska* & Jocelyn F. Bautista & Rolf Blauenfeldt \\
\hline Hugo J. Aparicio & Thomas G. Beach & Andrew Fabian \\
\hline Stephen Aradi & David Bearden & Bleasel \\
\hline Antonio Arauz & Martina Bebin & Andrew S. Blum \\
\hline Bhooma R. & Peter Bede & Dana Boebinger \\
\hline Aravamuthan & Edward M. & Christopher J. Boes \\
\hline Francesco Arba & Bednarczyk & Sylvia M. Boesch \\
\hline Giorgio Arcara & Danny Bega & Bradley F. Boeve \\
\hline Eider M. Arenaza- & Ettore Beghi & Julien Bogousslavsky \\
\hline Urquijo & Luca Bello & Leonardo Bonilha \\
\hline Juan F. Arenillas & Barbara Brigitta & Arturo Bonometti \\
\hline Zohar Argov & Bendlin & Per Borghammer \\
\hline Antonio Arjona & Lars Bendtsen & Hugo Botha \\
\hline Carmel Armon** & Giulia Benedetti & Mark I. Boulos \\
\hline Melissa J. & Bruno A. Benitez & Gregoire Boulouis \\
\hline Armstrong** & Julián Benito-Leon* & Dennis Bourdette ${ }^{* *}$ \\
\hline Georgina Arrambide & Elinor Ben- & Pierre Bourdillon \\
\hline Ethem Murat Arsava & Menachem & Mehdi Bouslama* \\
\hline Daniel Arteaga & David A. Bennett & James D. Bowen \\
\hline Ravindra Arya & Jeffrey L. Bennett & James H. Bower \\
\hline Kapil Arya & Wyatt P. Bensken* & Lara Boyd \\
\hline Masato Asahina & Anne Berberich & Geraldine B. Boylan \\
\hline Eishi Asano & Anne T. Berg** & Bernard C. Brais \\
\hline Alberto Ascherio & Joseph R. Berger & Marcus Vinicius \\
\hline Sharon Ash & Gregory K. Bergey & Branco de Oliveira \\
\hline Breton Michael & Niels Bergsland & Ethan S. Brandler \\
\hline Asken & Samuel F. Berkovic & John Frederick \\
\hline Masharip & Aaron L. Berkowitz ${ }^{*}$ & Brandsema* \\
\hline Atadzhanov & Madison M. Berl & Robynne Braun \\
\hline Bertrand Audoin & Raphael Bernard- & John C. Breitner* \\
\hline David S. Auerbach & Valnet & David Brenner \\
\hline Eitan Auriel & James L. Bernat* & J. Nicholas Brenton \\
\hline Stéphane Auvin & Julie Bernhardt & Marianthi Breza \\
\hline N. Ahmad Aziz* & James D. Berry & Farren Basil Shaw \\
\hline Neeraj Badjatia & Anna Bersano & Briggs \\
\hline Umesh A. Badrising & David Bervini & Francesco Brigo \\
\hline Sergio Bagnato & Anand Bery & Waleed Brinjikji \\
\hline
\end{tabular}

Benjamin $\mathrm{H}$.

Brinkmann

Tom Britton

Amy Brodtmann**

Mark B. Bromberg

Adolfo M. Bronstein

Sarah Marie

Brooker**

David James Brooks*

Matthijs C. Brouwer

Devin L. Brown*

Wallace Brownlee

Norbert

Brüggemann

Audrey Christine

Brumback

Nicola Brunetti-

Pierri

Oliviero Bruni

Iris Brunner

John C.M. Brust*

Maria Jose Bruzzone Giraldez*

Rachel F. Buckley**

Thomas A. Buckley

Joshua Amit Budhu

Andrew E. Budson

Dennis R. Buis

Krzysztof Bujarski

James F. Burke

David Bruce

Burkholder

Jorge G. Burneo

Joseph D. Burns**

Robyn M. Busch

Saif Bushnaq

Neil A. Busis

Nitin Butala

Russell J. Butterfield

Jaclyn B. Caccese

Peter A. Calabresi

Daniel Calame*

Aaron Caldwell

Pietro Caliandro

Brian C. Callaghan*

Andrea Calvo

Lisa Calvo

Peter R. Camfield

Richard M. Camicioli

Bruce C.V.

Campbell*

Laura Canafoglia

Stefano F. Cappa

Juan R. Carhuapoma

Francisco Javier

Carod-Artal

Joseph Carrera
Ian Carroll

Michael S.

Cartwright

Kaitlin Blackstone

Casaletto

Terrence Cascino

Richard J. Caselli

Ilaria Casetta

Barbara Casolla

Luis Castilla-Guerra

Pablo R. Castillo

Sergio A. Castillo-

Torres

Eleonora Catricalà

Carlo Cavaliere

Andrea Eugenio

Cavanna

Jennifer Cavitt

Fernando Cendes*

Bruno Censori

Lana M. Chahine

Lina Chalak

Colin Chalk

Ling Ling Chan

Jason J. Chang

Cherylee W.J. Chang

Bernard P. Chang

Michael J. Chao

Sherita N. Chapman

Smith

Declan T. Chard*

David Charles

Seemant Chaturvedi*

Umair Javaid

Chaudhary

Gerardo Chavira

Hernandez ${ }^{*}$

Patrick Chen*

Wei Chen

Zhibin Chen

Robert Chen

Kewei Chen

Shuai Chen

Timothy Chen

Xing Chen

Celia S. Chen

Bastian Cheng

Edmund Cheong

Neena Cherayil

Luisa Chiapparini

Adriano Chio*

Tracey A. Cho

Kelvin L. Chou*

Stephen

Chrzanowski

Min Kyung Chu

Catherine J. Chu 
Miguel Chuquilin

Bart Chwalisz*

Alfonso Ciccone

Maria Roberta Cilio

Molly Conn Cincotta

Mehmet N. Cizmeci

Jens Claassen

Daniel O. Claassen*

Kristl G. Claeys

David B. Clifford

Trevor Cline

Keith A. Coffman

Hugo Cogo-Moreira

Mark L. Cohen

Fleur Cohen Aubart

James G. Colebatch

Micheal P. Collins

Howard Colman

Erin Conrad

Robin A. Conwit

Mark J. Cook

Elizabeth A. Coon

David R. Cornblath

Nick Corriveau-

Lecavalier

Pietro Cortelli

Gianluca

Costamagna**

Fiona E. Costello*

Robert P. Cowan

Joyce A. Cramer

Amy Crepeau

Daniel Crespo

Claire J. Creutzfeldt

Susan R. Criswell

Anne H. Cross

Salvador Cruz-Flores

Esther Cubo

Arthur Cukiert

Antonio Culebras

Jeremy K. CutsforthGregory*

Gary R. Cutter*

Aman Dabir

Dominic D'Agostino

Eleonora Silvana

D'Ambrosio

Eyiyemisi Damisah

Jean-Francois

Dartigues

Sirwan K.L.

Darweesh

Rohit Das

Neil Datta

Hina Dave

Kathryn A. Davis

Thomas L. Davis
Gregory Scott Day**

Mamede de

Carvalho*

Gabriel R. de Freitas

Adam de Havenon

Frank-Erik de

Leeuw*

Andres De Leon-

Benedetti*

Felipe De Los Rios la Rosa

Gian Marco De

Marchis

Marianne de Visser

Linda de Vries

Jennifer L. Dearborn

Aman Deep

Gagan Deep

Ellen M. Deibert

Steven T. DeKosky

Teodoro Del Ser

Claire Delbridge

Candice Delcourt

Guillermo Delgado-

García** $^{*}$

Gabriele C. DeLuca

Tom den Heijer

Mariel Brooke

Deutsch

Davangere P.

Devanand

Orrin Devinsky*

Richard B. Dewey, Jr.

Feza Deymeer*

Rajinder K. Dhamija

Radhika Dhamija

Klodian Dhana*

Antonio Di Carlo

Massimiliano Di

Filippo

Vincenzo Di Lazzaro

Francesco Di

Lorenzo*

Daniel Garbin Di

Luca*

David Diaz

Martin Dichgans

Nico Jean Diederich

Jose Danilo Bengzon

Diestro

Marianne Dieterich

Jorg Dietrich

Jacopo C.

DiFrancesco

Dale Ding

Ding Ding

Marc Dinkin
Afshin A. Divani

Nomazulu Dlamini

Dennis J. Dlugos

Bruce H. Dobkin

Tomas Dobrocky

Ruth Dobson

Hiroko H. Dodge

James D. Dolbow

Moises Dominguez

Rogelio Dominguez

Moreno

Lisanne

Dommershuijsen

Junling Dong*

Geoffrey Alan Donnan

Jan Dörr

Richard L. Doty

Fergus Doubal

Dar Dowlatshahi

James J. Dowling*

Daniel B. Drachman

Frank W. Drislane

Rose Du

Richard M.

Dubinsky**

Emma Duerden

Marco Duering

Kevin Duff

Carole Dufouil

Mario F. Dulay

John S. Duncan

Nicolas Dupre

P. James B. Dyck

Braydon Dymm

Tomasz Dziedzic

Rainer Dziewas

K.P. Ebmeier*

Steven D. Edland

Brian L. Edlow

Matthew Edwardson

Alexandra Eid

Andrew A. Eisen

Rodger J. Elble

Merrill F. Elias

Mitchell S.V. Elkind

Colin A. Ellis

Bakri H. Elsheikh

Jennifer Emond

Christian Endisch

Erik R. Ensrud

Arman Eshaghi**

Nima Etminan

Mahyar Etminan

Elisa Evangelista

Amelia Evoli

Tobias Djamsched

Faizy
Guido J. Falcone

John Y. Fang

Michael Fara

Nathan Farkas**

Michelle E. Farrell

Alex Thomas Farris

Alfonso Fasano*

Klaus Fassbender

Raymond Edward

Faught Jr.

Erin Fedak

Romanowski

Paolo Federico

Dominic B. Fee

Michael G. Fehlings

Emily Feneberg

Luigi Ferini-Strambi

Tanis J. Ferman

Juan Diego

Fernandez

Eduardo Fernandez

Nicola Ferrara

Julia Ferrari

Raffaele Ferri

José M. Ferro

Jeanne Feuerstein*

Terry D. Fife

Matteo Figini

Massimo Filippi**

Christopher M. Filley

Elizabeth Finger

Alan G. Finkel

Mark J. Fisher

Eoin P. Flanagan

Jori E. Fleisher

Evan Fletcher

Leon Flicker

Christian Foerch

Christophe Maxime

Fokoua Dongmo

Ana Catarina

Fonseca

Gary A. Ford

Andria L. Ford

Emma Foster

Christina N.

Fournier*

Robert J. Fox*

Christine K. Fox

Samuel A. Frank

Gary M. Franklin**

Birgit Frauscher

Daniel A. Freedman

Miriam L. Freimer

Jacqueline French

Daniel Friedman

Joseph H. Friedman*
Sarah Friedman

Benjamin W.

Friedman

Giovanni B. Frisoni

Karl Friston

Richard Eugene Frye

Katherine A. Fu**

Jennifer E. Fugate

Jong-Ling Fuh

Shinsuke Fujioka

Hidenao Fukuyama

Timothy Fullam

Julio C. Furlan

Ansgar J. Furst

Julia Furtner

Satish Gaddam

Gloria Galloway

Javier Andrés

Galnares-Olalde

Marian Galovic

Charlene E. Gamaldo

Carlo Gandolfo

Aravind Ganesh

Vijay Ganesh

Mary Ganguli

Gautam Ganguly

Taneeta Mindy

Ganguly

Dawn Gano

Ziv Gan-Or

Paul A. Garcia

Rocio Carolina

Garcia-Santibanez

Aayushi Garg

Valentina Garibotto

Peter Garrard

Sara Gasparini

Thomas Gasser

Thomas Gattringer

Maiya R. Geddes

Jeffrey Marc

Gelfand

Matthew Gentry

Marios K. Georgakis

Benjamin P. George

Ilena George*

Elizabeth E. Gerard

Christian Gericke*

Angelo Ghezzi

Christopher $\mathrm{H}$. Gibbons

Donald L. Gilbert

Gordon J. Gilbert

Nils Erik Gilhus

Chris Gillette

Emily Gilmore

Wendy Gilmore 


Andrea Gilmore-
Bykovskyi
Paola Gilsanz
Jack Gladstein
Jonathan D. Glass
Torin Glass
Ezequiel
Gleichgerrcht
David Gloss**
M. Maria Glymour**
Simon Glynn
Peter J. Goadsby
Florent René
Maurice Gobert
Christopher G.
Goetz
Daniel Gold
James N. Goldenberg
Daniel M.

Goldenholz

Alica M. Goldman*

Larry B. Goldstein*

Grace Yoonheekim Gombolay

David Gomez Andres

John Gommans

Francisco de Assis

Aquino Gondim

Julie Gonneaud

Nicole R. Gonzales*

Mitzi M. Gonzales

Luis Nicolas

Gonzalez Castro

Brian Andrew

Gordon

James M. Gordon

Philip B. Gorelick

Kenneth C. Gorson

Shweta Goswami

Jean Gotman

Satoshi Goto

Rebecca F.

Gottesman*

Christopher

Gottschalk

Gaurav Goyal

Mayank Goyal

Jerome J. Graber*

David Grabli

William D. Graf

Tobias Granberg

Claudia A.

Granbichler

Mark A. Granner

Cristina Granziera

Linda Gray

Alexander L. Green
Kemar E. Green

Steven A. Greenberg

Steven M. Greenberg

Beverly Greenspan*

Robert S.

Greenwood

Robert C. Griggs

Timo Grimmer

Zachary M. Grinspan

Wolfgang Grisold*

Rob J.M. Groen

Scott Grossman

James C. Grotta

Aashrai Sai Venkat

Gudlavalleti

Zain Guduru

Adrien Guenego

Renzo Guerrini*

Jiang Gui

Amanda C. Guidon*

Kristin Guilliams

Edwin Gulko

Himanshu Gupta

Deepak K. Gupta

Gita Gupta

Harsh Vardhan

Gupta

Jeff Guptill

M. Edip Gurol

Deborah Gustafson

Jose Gutierrez

David H. Gutmann

Laurie Gutmann

Amy Katherine

Guzik

Yael Hacohen*

Manji Hadi

Timothy C. Hain

Oday Halhouli

Ara S. Hall

Gabor Michael

Halmagyi

Casey H. Halpern

Steffen Hamann

Marla J. Hamberger

Ali G. Hamedani

Hajo M. Hamer

Katherine T.

Hamilton

Roland T. Hamilton

Takashi Hanakawa

Thomas Folkmann

Hansen

Stephen T. Hantus

Ihtsham U. Haq

Orla Hardiman

Noam Y. Harel*
Matthew Harmelink

Matthew Harms

Judith U. Harrer

Adil Harroud

Robert G. Hart

Adam L. Hartman*

Danielle Harvey

Anhar Hassan

Jason Hassenstab

Taku Hatano

W. Allen Hauser

Sheryl R. Haut

Fiona Hawke

Xiaosong $\mathrm{He}$

E. Tessa Hedley-

Whyte

Kristen Heinan

Kerstin Hellwig

Suzanne Hendrix

JoonNyung Heo

Isabella Herman**

Bruce Hermann

David N. Herrmann

Andrew D. Hershey

Andrew G. Herzog

Geoffrey L. Heyer*

Daniel B. Hier

Saima Hilal

Chloe E. Hill

Christopher Joseph

Hill

Susan L. Hillier

Argye Elizabeth

Hillis

Michio Hirano

Teruyuki Hirano

Georg Hishaw

Lisa D. Hobson-

Webb

Matthew T. Hoerth

Eric P. Hoffman

Jan Hoffmann

R. Edward Hogan

Eva Hogervorst

Birgit Högl

Gunter U. Hoglinger

Samantha K. Holden

J. Lloyd Holder, Jr.

Matthias Holdhoff

Robert G. Holloway

Trygve Holmoy

R.M. Damian

Holsinger

Martin Holtkamp

Jennifer Hong

Babak Hooshmand*

Jennifer L. Hopp
Andreas Hottinger

Virginia J. Howard

Jennifer Hranilovich

Amie W. Hsia

Sung-Tsang Hsieh

Le H. Hua

Jason H. Huang

Katherine C. Hughes

Hanneke E. Hulst

Ruth Huna-Baron

Rachael Maree

Hunter**

Robert Hurford**

Aatif M. Husain

Shaun A. Hussain

Michael Hutchinson

David Y. Hwang

Susan T. Iannaccone

Rebecca N. Ichord

Nneka Lotea Ifejika

Katrina Hannah D. Ignacio**

Masafumi Ihara

Takahiro Iizuka

Kachi Illoh

Yuichiro Inatomi

Caroline Ingre

Sarosh R. Irani

Pablo Irimia

David John Irwin*

Stefan Isenmann

Jean Isnard

Autumn Ivy

Fabio M. Iwamoto

Juha E. Jääskeläinen

Michael Jacewicz

Giris Jacob

Daniel H. Jacobs

Bradley S. Jacobs

Michael S. Jaffee

William J. Jagust

Klaus Jahn

Assia Jaillard*

Bryan David James

Michael L. James

Todd Janus

Valerie Jeanneret

Joanna C. Jen

Johann Malte Enno Jende

Thomas M. Jenkins

Xia Jiang

Glen Jickling**

Hyder Azad Jinnah

Michelle C. Johansen

Sterling C. Johnson

Derek R. Johnson
Lyell K. Jones*

Lori C. Jordan*

Justin T. Jordan*

Raed A. Joundi

Eric Jouvent

Ralph F. Jozefowicz

Suzanne E. Judd

Vern C. Juel

Csaba Juhasz

Larry Junck*

Jinsei Jung

Arash Kahrom

Rajesh N. Kalaria

Tomas Kalincik

Bernadette Kalman

Sanjay Kalra

Stephen S. Kamin

Henry J. Kaminski

Margitta T.

Kampman

Joseph Kamtchum Tatuene*

Nagaendran Kandiah

Vinay Vardhan

Reddy Kandula

Peter B. Kang**

George T. Kannarkat

Ronald M. Kanner

Hideyuki Kano

Petr Kanovsky*

Amit Kansara

Gregory Kapinos*

Peter W. Kaplan

Moira K. Kapral

Amel Karaa

Ioannis Karakis

Chafic Karam*

Ashwin Karanam

Jason Karlawish

Matthias Karst

Edward J. Kasarskis

Carlos S. Kase

Gregor Kasprian

Meike S. Kasten

Brittany M.

Kasturiarachi

Mira Katan*

Gaurav Kathuria*

Mitsuhiro Kato

Aristeidis $\mathrm{H}$.

Katsanos*

Jorge C. Kattah*

Jonathan S. Katz

Keisuke Kawata

Mark Robert Keezer

Gordon R. Kelley

Adam Kelly 
Dearbhla Kelly

Kimbra Kenney*

Kevin A. Kerber

Kyle Carson Kern

Wesley T. Kerr*

Navin Kesari*

Tigran Kesayan

Zafer Keser

Sudha Kilaru Kessler

Yasmin Khakoo

Raja B. Khan

Imad Khan

Pouya Khankhanian

Charles J. Kidd*

Desmond Patrick Kidd

Laura L. Kilarski

Joep Killestein*

Ronald J. Killiany

Ho Jin Kim

Beom Joon Kim

Han-Joon Kim

Ji-Soo Kim

Sung-Min Kim

Teresa J. Kimberley

W. Taylor Kimberly

Michael O. Kinney

Ilka Kleffner

Christopher J. Klein*

Andrea Klein

Pavel Klein

Matthias Klein

Brad C. Klein

Christoph

Kleinschnitz

Laura Klesse

Kelly Knupp

Eliane Kobayashi

Marcus Werner Koch

Arnulf H. Koeppen

Susan Koh

Wolfgang Köhler

Alfried Kohlschütter

Akio Koizumi

Noah Allan Kolb

Shannon Kolind

Brad Kolls

Dennis Kolson

Katie Kompoliti

Barbara S. Koppel

Pearce J. Korb

Amos D. Korczyn

Miikka Korja*

David N. Korones

Vladimir S. Kostic

Vikas Kotagal

Katarzyna Kotulska
Prateeka Koul

Alain K. Koyama*

Neha M. Kramer

Gregory L. Krauss

Hubertus Kremer

Christos Krogias

Michael C. Kruer

Lauren B. Krupp

Kristen M. Krysko

Jens Kuhle

Wilhelm Kuker

Walter A. Kukull

Erin R. Kulick

Jaime Kulisevsky

Lewis H. Kuller

Kunal Kumar

Nancy Kuntz

Wolfram S. Kunz

Sheng-Han Kuo

Naoto Kuroda

Tobias Kurth

Angelo Labate

David M. Labiner

Brittany Bolduc

Lachance

Deepak Krishan

Lachhwani

Catherine C. Lacroix

Kathrin LaFaver

Robert Laforce, Jr.

Alfonso Lagares

Eugene C. Lai

Keng Lam*

Herm J. Lamberink

Isabelle Lambert

Gert Jan Lammers

Guillaume Lamotte

Christian Lampl

Susan Landau

Doriana Landi

Michael Alexander

Lane

Douglas R. Langbehn

Douglas J. Lanska

Michel P. Lanteri-

Minet

Giuseppe Lanza

Giuseppe Lanzino

Susanna C. Larsson

Julius Gene Silva

Latorre* $^{*}$

Larry Latour

John A. Lawson

Steven M. Lazar*

Ronald M. Lazar

Victoria M.

Leavitt**
Christine Lebrun-

Frenay

John J. Leddy

Mark S. LeDoux**

Andrew Lee

Mi Ji Lee

Jong Woo Lee

Stephane Legriel

Richard Leigh

Enrique C. Leira*

Alain Lekoubou

Abhishek Lenka*

Gaetan Lesca

Thabele M. Leslie-

Mazwi

Sue E. Leurgans*

Kerry H. Levin

Steven R. Levine

Michael Levitt

Michael Levy

Gilberto Levy

Richard A. Lewis

Frank Leypoldt

Didier Leys

Cristian E. Leyton

Wenyuan Li

Yi Li

Linxin Li

Yingkai Li

David G. Lichter

Frederique Liegeois

Thomas Lillicrap

Shen-Yang Lim

Michelle P. Lin*

Jia Lin*

Jenny Lin

Chin Lon Lin

Antti E. Lindgren

Anita Lindmark

Hester F. Lingsma

Vasileios-Arsenios

Lioutas

Richard B. Lipton*

Robert P. Lisak

Feng Liu

Yin Allison Liu

Yanhui Liu

Xinfeng Liu

Alberto Lleo**

Rafael Llinas

Warren D. Lo*

Raymond Y. Lo

Benjamin W.Y. Lo

David A.

Loewenstein

Giancarlo

Logroscino
Zachary London

Iscia Lopes-Cendes

Oscar L. Lopez

Alfonso Sebastian

Lopez-Chiriboga*

Svetlana Lorenzano*

Jian-Qiang Lu

Xiangfeng $\mathrm{Lu}$

Marie L. Luby

Gert-Jan Luijckx

Dorothée E. Lulé

Ingrid Lundberg

Michael Lunn

Dana Lustbader

Andrew L. Lux

Donald Lyall

Michael J. Lyerly

Timothy Lynch

Ariel M. Lyons-

Warren*

Matthew Brandon

Maas

E. Anne MacGregor

Judith Machts

Mary Machulda

Carolina Barbosa

Maciel

Kenneth J. Mack

Tracy E. Madsen*

Alain Maertens de

Noordhout

David Magnus

Uma Mahajan

Luis F. Maia

Jennifer Juhl

Majersik*

Margaret K.Y. Mak

Angela M. Malek

Paresh A. Malhotra

Rainer Malik

Eric J. Mallack

Robert M. Mallery

Chindo Bala Mallum

Andrew L. Mammen

Ashutosh K.

Mangalam

Sara Manning

Tomoo Mano

Ali Manouchehrinia

Georgios

Manousakis

Sneha Mantri

Javier Mar

Romain Marignier

Sara Mariotto

Patrick Mark

Niklas Marklund
Mirketa Marku

Wilson Marques

Juan Manuel

Marquez-Romero

Connie Marras

Ruth Ann Marrie

Jennifer Marsella

Elisabeth Breese

Marsh

Eric D. Marsh

Randolph S.

Marshall $^{*}$

Wayne Martin

Davide Martino

Andrea Martinuzzi

Sina Marzoughi

Sarah L. Mason

Shavonne Massey

Colin L. Masters

David B. Matchar

Farrah J. Mateen

Gary W. Mathern

Makoto Matsui

Emma L. Matthews

Anna Matynia

Alberto Maud

Michael Mazya

Anna Teresa Mazzeo

Patrick F. McArdle

Justin C. McArthur

Lauren McCollum*

Eric McDade

Paul D. McGeoch

Scott M. McGinnis

Corey McGraw

Joseph F. McGuire

Christopher

McLouth

Hugh J. McMillan*

Patrick McQuillen

Simon Mead*

Kimford J.

Meador**

Mark F. Mehler

Prachi Mehndiratta

Tapan Mehta

Shyamal H. Mehta

Alexandria Ellen

Melendez-Zaidi**

Stefano Meletti

Susanna Melkas

Jerry R. Mendell

Giovanni Meola

Jose Miguel Laffita

Mesa

Walter Steven

Metzer* 
Mónica Beatriz

Mezmezian

Rosa Michaelis

Michelle M. Mielke*

Raffaella Migliaccio

John W. Miller*

Steven P. Miller

Rebecca K. Miller-

Kuhlmann

Colleen Mills-

Finnerty

Berge A. Minassian

Mia T. Minen

Jonathan W. Mink

Mark Mintz

Pablo Mir

Ario Mirian*

Shubhankar Mishra*

Nishant K. Mishra

Eva A. Mistry

Wendy G. Mitchell

Michael Mlynash

Alisa $\mathrm{Mo}^{* *}$

Pedro J. Modrego

Jeremy J. Moeller*

Abhay Moghekar

Fatemeh

Mohamamdpour

Touserkani

Shekeeb S.

Mohammad

Nimish A. Mohile

Xavier Moisset

Jeremy A. Molad

Annette Molinaro

Mauro Monforte

Gabriele Monte

Jacqueline Montes

Majaz Moonis

Karla A. Mora

Rodriguez

Lewis B.

Morgenstern

Germán Morís

Elizabeth Mormino

Elena Moro

Andrea Morotti

Martha J. Morrell

Nicholas A. Morris*

James A. Mortimer

Solomon L. Moshe

Tahseen Mozaffar*

Lorenzo Muccioli*

Susanne

Muehlschlegel

Christoph Mueller

Nils Muhlert*
Wolfgang G.

Muhlhofer

Ryan Thomas Muir*

Marco Mula

Kassandra Munger

Heidi Munger Clary

David G. Munoz

Paolo A. Muraro

Melissa E. Murray

Santosh B. Murthy

Martin Mwangi*

Kenneth Alexis

Myers

Krishna Mylavarapu

John Mytinger

Benedetta Nacmias

Zurab Nadareishvili

Elie Naddaf

Stephen E. Nadeau

Yoshinari Nagakane

Nandakumar

Nagaraja

Olivier Naggara

Dileep R. Nair

Kaavya Narasimhalu

Pushpa

Narayanaswami*

Fábio Augusto

Nascimento**

Cody Nathan

Roland Nau

Xavier Navarro

Merrilee Needham

Ahmad Nehme*

Maromi Nei

Aidan Neligan

Bryan J. Neth

Hermann

Neugebauer

Bernhard Neumann

Rinze Frederik

Neuteboom

Kathryn Nevel

Joel Neves Briard**

Douglas E. Ney

Yu-Tze Ng

Tiia Ngandu

Joseph Ngeh

Thanh N. Nguyen

Jennifer M. Nicholas

Fenwick T. Nichols

Katharine Ames

Nicholson

Miriam Nickel

N. Nighoghossian

Ichizo Nishino

Flavio Nobili
Michael J. Noetzel

Yoshiko Nomura

Gina Norato

Anna Starikovsky

Nordvig

Jonathan A. Norton

George Ntaios

Adam Lawrence

Numis

John G. Nutt

Lars Nyberg

Paul A. Nyquist

Saskia A.J. Lesnik

Oberstein

Terence John

O’Brien

Finbar O'Callaghan

Cumara O'Carroll*

Nate Sean O'Connell

Yazmin Odia

Nicole Odom

Steve O’Donnell

Cormac A.

O’Donovan

Akinkunmi

Okekunle

Susanna S. O’Kula

Mark A. Oldham

Fabricio Ferreira de

Oliveira

Jean Marc Olivot

DaiWai M. Olson

Charlene Ong

Osamu Onodera

Marco Onofrj

Daniel Ontaneda

Serena L. Orr

Benjamin Osborne

Maryam Oskoui

Rik Ossenkoppele

Adam P. Ostendorf

Patrick Ostkamp

Padraig

O’Suilleabhain

Brian R. Ott

Genko Oyama

Tetsutaro Ozawa

Lisa M. Pabst

Andrea Pace

Nicholas M. Pajewski

Lina Palaiodimou

Raffaele Palladino

Jose-Alberto Palma

Andre Palmini

Sebastian Palmqvist

Priya Palta

Jie Pan $^{*}$
V. Shane Pankratz ${ }^{* *}$

Leonardo Pantoni**

Francesco Panza

Constantinos

Papadopoulos

Piero Parchi

Melissa Shuman

Paretsky*

Davide Pareyson

Maksim Parfyonov

Gunjan Parikh

Neal S. Parikh

Hyung Jun Park

Lucilla Parnetti

Markku Partinen

Matthew Paul Pase*

Pau Pastor*

Maria A. Pastor

Archana A. Patel

Arpan Patel*

Anup D. Patel

Ross W. Paterson

Sheel Pathak

Jorge E. Patino Murillas

Robert H. Paul

Steven G. Pavlakis

Valory N. Pavlik

Phillip L. Pearl

Toni Pearson

José Luiz Pedroso

Victoria Susan Pelak

Maria Teresa

Pellecchia

Sarah T. Pendlebury

Patricia E. Penovich

Umberto Pensato*

Katell Peoc'h

Joanna Pera

Rafael Perera

David L. Perez

Joel S. Perlmutter*

M. Scott Perry

Nils Peters

Ronald C. Petersen

Corinne Pettigrew

Jeffrey Phillips

John D. Pickard

Fredrik Piehl

Fabio Pilato

Iago Pinal-Fernandez

Pranusha Pinna

Marcus V. Pinto*

Amanda Piquet

Geoffrey Pitt

Sean J. Pittock

Peggy J. Planetta**
Giuseppe Plazzi

David Pleasure

Anna Poggesi

Heiko Pohl

Sven Poli

Luisa Politano

Thomas Pollmacher

Bruce E. Pollock

Octavio Marques

Pontes-Neto

Aurel Popa-Wagner

Brenda E. Porter

Alyx B. Porter

Jose Posas

Cyril Pottier

William J. Powers

Pauli Pöyhönen

Ferran Prados

Craig Adam Press

Peter S. Pressman

David C. Preston

Tamara M.

Pringsheim*

Alberto Priori

Michael Privitera

Calin I. Prodan

Luca Prosperini

Federica Provini

Klearchos

Psychogios

Francesca Puledda

Francisco F. Purroy

Jukka Putaala

Nataliya Pyatka

Romana Pylypchuk

Aldo Quattrone

Luis Querol

Mark Quigg

Terence J. Quinn

Imran H. Quraishi

Jacob Raber

Jennifer Rabin

Alejandro A.

Rabinstein

Sara Radmard

Alex D. Rae-Grant

Alberto Raggi

Ann B. Ragin

Zebunnessa Z.

Rahman

Jidhin Raj

Shruti Raja

Yusuf A. Rajabally

Ali H. Rajput

Alexander H. Rajput

Ramnath Santosh

Ramanathan 
Sudarshini

Ramanathan

Ciro Ramos-

Estebanez

Anna Ranta

Jaivir S. Rathore

Marcia Hillary

Ratner

Radoslav I. Raychev

Sumanth Reddy

Anthony T. Reder

Jens Peter Reese

Stephen G. Reich

Evan Reid

Mary M. Reilly

Jared P. Reis

Norman R. Relkin

Leonardo Renieri

Susan M. Resnick

Lucas Restrepo

Kathryn M. Rexrode

Harmen Reyngoudt

Chandra A. Reynolds

Nosheen Reza

Michael Rezak

Michael Reznik

Sylvain Rheims

Raffaella Ricci

Nina Y. Riggins

E. Bernd Ringelstein

John M. Ringman

John Robert Rinker

Bastien Rioux*

Pamela M. Rist

Christine Seel

Ritchie

Lucia Rivera-Lara

Giovanni Rizzo

William B. Rizzo

Benjamin B. Roa

Jennifer Robblee

Jodie I. Roberts**

Maisha T. Robinson

Jessica Robinson-

Papp

Maria A. Rocca*

Raquel Rocha*

Stefanie Rodenbeck

Robert L. Rodnitzky

Roberto Rodríguez

Rivas*

Shanu F. Roemer

Leonardo Roever

Julio C. Rojas

Alberto Romagnolo

Gustavo C. Roman

Jose G. Romano
Gabriel M. Ronen

Steven N. Roper

Caterina Rosano

Anna Rosati

Cristina Rosazza

Paul B. Rosenberg

Gary A. Rosenberg

David B. Rosenfield

Eric Scott

Rosenthal

Elliot D. Ross

Paolo M. Rossini

Andrea L. Rosso

Natalia S. Rost

Elliot J. Roth

Aaron Rothstein*

Dalia Rotstein

Alex Rovira

Bhaskar Roy

Emile P. Roy

Anna Rubegni

Theodor Rüber

Anthony Rudd

Steven Rudolph

Stephan J. Rüegg

Alessandra Rufa

Robert L. Ruff

Ynte M. Ruigrok

Jeffrey Russ**

Antonio Russo

Loes C. A. Rutten-

Jacobs

Philippe Ryvlin

Mario Sabatelli

Maria Sabater-Lleal

Behnam Sabayan*

Marwan N. Sabbagh

Hamidreza Saber

Simona Sacco

Saud A. Sadiq

Reza Sadjadi

Joseph E. Safdieh

Ram Sagar

Ali A. Saherwala

Marie-Helene Saint-

Hilaire

Martha Sajatovic

Kalyan Sajja

Mohammad Kian

Salajegheh

Stephen P. Salloway

Amber Salter ${ }^{* *}$

Debopam Samanta

Rohini D.

Samudralwar

Christa O’Hana V.

San Luis
Iván Sánchez

Fernández

Josemir Sander*

Paola Sandroni

Tristan Timothy

Sands

Else Charlotte

Sandset

Nerses Sanossian

Valeria A. Sansone

David S. Saperstein

Vincent Sapin

Fred S. Sarfo

Muralidharan

Sargurupremraj

Alessia Sarica

Harini Sarva

Aarti Sarwal*

Douglas Kazutoshi

Sato

Neda Sattarnezhad

Justin A. Sattin

Dorothee Saur

Rodolfo Savica*

Stephen J. Sawcer

Deanna R. Saylor

Nikolaos Scarmeas

Stephen N. Scelsa

Steven C. Schachter

Christof Schaefer

Ulrike Schara-

Schmidt

Ingrid E. Scheffer

Carlos H. Schenck

Ann I. Scher

Nicoline Schiess

David Schiff*

Stephanie

Schipmann

Helena Schmidt

Michal Schnaider

Beeri* $^{*}$

Caroline Schnakers

Michael Joel Schneck

Hauke Schneider

Logan Douglas

Schneider

Andrea Lauren

Christman

Schneider

Julie A. Schneider

Jordana S. Schneider

Michael Schöll

Menno M.

Schoonheim*

Nina F. Schor

Benedikt Schoser
Jonathan M. Schott

John M. Schreiber

Stephan U. Schuele

Norbert Schuff

Jan M. Schwab

Jason M. Schwalb

Heidi Beck Schwarz

Angelo Scuteri

Pavel Seeman

Kaitlin Seibert

Michael Seidenberg

David Julian Seiffge*

John B. Selhorst

Olga Selioutski

Johann Sellner

Souvik Sen

Arjune Sen

Sang Won Seo

Esra Serdaroglu*

Alberto Serrano-

Pozo

Laurent Servais

Serenella Servidei

Adonis Sfera

Amir Shaban

Yash D. Shah*

Vishank Arun Shah

Suma Shah

Pashtun Shahim

Rohan Sharma*

Vijay K. Sharma

Jeremy M. Shefner

Kevin Navin Sheth

Jiong Shi

Holly Shill

Hitoshi Shinotoh**

Liqi Shu

Saurabh G. Shukla**

Lori Shutter

Michael E. Shy

Gull Zareen Khan

Sial

Christos

Sidiropoulos

James E. Siegler

Alessio Signori

Michael H. Silber

Robert Silbergleit

Gisele Sampaio Silva

Laura Silveira-

Moriyama

Brian Silver

Julie K. Silver

Mauro Silvestrini

Neil G. Simon

Michela Simoni

Claus Z. Simonsen
Kristina Simonyan

Alexis N. Simpkins

David M. Simpson

Tanya Simuni

Mamta Bhushan

Singh

John Robinson

Singleton

Saurabh R. Sinha

Antonio Siniscalchi

Nils Skajaa

Ingmar Skoog

Jane Skov

Eric E. Smith*

Wade Smith

Mary Lou Smith

A. Gordon Smith

Garnett Smith

Todd A. Smitherman

Beth Ellen Snitz*

Julie S. Snowden

Amna Sohail*

Salil Soman

Yinchen Song

Maria Pia Sormani**

Rebecca I. Spain

Gianfranco Spalletta

Nicole L. Spartano

Roderick Spears

Andrew R. Spector

Jaime Lynn Speiser**

J. David Spence

Luciano A. Sposato*

Neil James Spratt

Jaiashre Sridhar

Erik K. St. Louis

Richard Staba

William Stacey

Michael P.H. Stanley

Hermann Stefan

Egon Stenager

Werner Stenzel

Brian D. Stephens

Yaakov Stern

Barney J. Stern

Walter F. Stewart

Jon Stone

Daniel Strbian

Jonathan Y. Streifler

Pasquale Striano

Eva M.M. Strijbis

Aaron F. Struck

Michael Strupp

Adam Strzelczyk

Olaf Stuve

S.H. Subramony

Lisa Renee Sun 


\begin{tabular}{|c|c|c|c|c|}
\hline Daokun Sun & Oliver Tobin & Sara Van Mossevelde & Bianca Weinstock- & Courtney J. \\
\hline Gene Sung & Benjamin David & Paul C. Van Ness & Guttman & Wusthoff* \\
\hline N. Abimbola & Tolchin & Susanne J. van Veluw & Sandra Weintraub & Thomas Wychowski \\
\hline Sunmonu & John B. Toledo & Nicola Vanacore & Michael D. Weiss & Michael L. Wynn* \\
\hline Katharina Stibrant & Torbjörn Tomson & Wim Vandenberghe & Michael Weller & Zongqi Xia \\
\hline Sunnerhagen & Silvina Beatriz & Panayiotis N. Varelas & Richard Wennberg & Denise J. Xu** \\
\hline Heidi Sutherland & Tonarelli & John M. Varlotto & John Kent Werner & Yasumasa Yamamoto \\
\hline Lukas Sveikata & Ahmed T. Toosy & Hartmut Vatter & David John Werring & Ryo Yamasaki \\
\hline Anders Svenningsson & Mehmet Akif & Aravindhan & Mary Ann Werz & Michele L. Yang \\
\hline Michael Swash & Topcuoglu & Veerapandiyan* & Lawrence J. Whalley & Dixon Yang \\
\hline Jeffrey Alan Switzer & James C. Torner & Michele Veldsman & Anne Wheeler & Sevil Yasar \\
\hline Minoru Tagawa & Gabriel Torrealba & Raghu Vemuganti & John R. Wherrett & Paul A. Yates \\
\hline Ryosuke Takahashi & Acosta & Charles Venuto & Michael D. White & E. Ann Yeh** \\
\hline Makoto Takahashi & Jose Torres & Jennifer Vermilion & Jennifer L. Whitwell & Wei Zhen Yeh \\
\hline Daiki Takewaki & Kazunori Toyoda & Steven Vernino & Meredith Wicklund & Nilufer Yesilot \\
\hline Daniel Talmasov** & Joseph I. Tracy & Meike W. Vernooij & Peter Widdess-Walsh & W. Katherine Yih \\
\hline Arturo Tamayo & Christopher B. Traner & Jan J. Verschuuren & Elysa Widjaja & Tomokatsu Yoshida \\
\hline Can Ozan Tan & Fatima Traore & Marie Vidailhet & Samuel Wiebe & Sohei Yoshimura \\
\hline Eng King Tan* & Helen Tremlett & Robert A. Vierkant & Heinz Wiendl* & Mitsuhiro Yoshita \\
\hline Bee Tan & Edwin Trevathan & Marta Vila-Pueyo & Gerald J. Wiest & Michael J. Young \\
\hline Christy C. Tangney & Elina Tripoliti & Tiago Villanuev & Sarah Wiethoff & William B. Young \\
\hline David F. Tang-Wai & Lynn Marie Trotti & Pablo Villoslada & Cami & k Yuan \\
\hline Carmela Tartaglia & Jack W. Tsao & Francois J.G. & Wijngaarde & Craig M. Zaidman \\
\hline Laura Tassi & Georgios Tsivgoulis & Vingerhoets & Donna M. Wilcock & Michael S. Zandi \\
\hline Yohei Tateishi & Amy R. Tso & Carlo Fiore Viscomi & Edward J. Wild & Ana Luiza Zaninotto \\
\hline Turgut Tatlisumak & Shoji Tsuji & David Viskochil & Einar P.V. Wilder- & Alonso Gonzalo Zea \\
\hline Myriam Taverna & Han Tun & George S. Vlachos & Smith & Vera $^{* *}$ \\
\hline Lynne P. Taylor & Carmen Tur* & Jacob W. Vogel & Barbara Willekens & Zecavati \\
\hline Matthew Tenan & Raymond Scott & Paula Emanuela & Auriel Willette & Helene Zephir \\
\hline Luigi Tesio & Turner & Voinescu & Joshua Zebadiah & Henrik Zetterberg \\
\hline Vijay M. Thadani & Grace M. Turner & Bruce T. Volpe & Willey & Chen Zhao \\
\hline Roshni Thakkar & Martin R. Turner & Rhonda R. Voskuhl & Shawr & Chongke Zhong \\
\hline Smathorn & Ken Uchino & Brad Voytek & & Zhu \\
\hline Thakolwiboon* & Ryan J. Uitti & Chris Vriend & Michael A. Williams & Jay-Jiguang Zhu \\
\hline Mangayarkarasi & Robin Ulep & Anh-Thu Ngo Vu & Robert S. Wilson* & Andreas Ziegler \\
\hline Thandampallayam & Toshitaka Umemura & Steve Vucic & Michael R. Wilson & Peyman Zirak \\
\hline Sujata P. Thawani & David K. Urion & Sandra Vukusic & Elaine C. Wirrell & Sasha Zivkovic \\
\hline Simon Thebault & Makoto Urushitani & Manav V. Vyas & Adina Wise ${ }^{* *}$ & Nathan T. \\
\hline William H. Theodore & Gitendra Uswatte & Ryan G. Wagner & Jens Witsch* & Zwagerman \\
\hline Ruth Thiex & Maarten & Tobias Walbert & Karsten Witt & Those who have \\
\hline Vincent N. Thijs & Uyttenboogaart & Amy Tara Waldman & Veronica Witte & participated in the \\
\hline Liu Lin Thio & Aparna Vaddiparti & Keenan A. Walker & Max Wiznitzer & Neurology ${ }^{\circledR}$ \\
\hline Robert Joseph & Farhaan S. Vahidy* & Asya Izraelit Wallach & Yohannes Woubishet & Podcast or \\
\hline Thomas & David E. Vaillancourt & Mitchell T. Wallin & W. Woldeamanuel & Neurology Minute $^{\circledR}$ \\
\hline George P. Thomas & Andrei A. Vakhtin & Kyle Walsh & Nicole I. Wolf* & are recognized \\
\hline David M. Thompson & Ignacio Valencia & Miranda Wan* & Charles Wolfe & below. \\
\hline Robert Thompson & Philip Van Damme & Tian Wang & Gil I. Wolfe & Justin Abbatemarco, \\
\hline Stone & Wiesje M. Van der & Annabel Kim Wang & Sae-Yeon Won & $\mathrm{MD}$ \\
\hline Amanda G. Thrift & Flier & Jian Wang & Ian R. Woodcock & Halley Briglia \\
\hline Majda M. Thurnher & Marjo S. van der & Cynthia X. Wang & Matthew Ryan & Alexander, MD \\
\hline Terrence Y. Tian & Knaap & Zhenchang Wang & Woodward* & Rae Bacharach, DO \\
\hline Steffen Tiedt & Ingrid A. van der Mei & Nick S. Ward & Clinton B. & Matthew Barrett, \\
\hline Ann Henderson & Thilo Van Eimeren & Joanna M. Wardlaw & Wright & $\mathrm{MD}, \mathrm{MSc}$ \\
\hline Tilton & Ellis S. van Etten & Katja E. Wartenberg & Peter Wrigley & Jennifer Bickel, MD, \\
\hline Dagmar Timmann & Jay A. van Gerpen* & Hirohisa Watanabe & Gregory Wu & FAAN, MAHS \\
\hline Mar Tintore & Argonde Corien van & Patrick J. Waters & Allan Wu & Christopher Boes, \\
\hline Maarten J. Titulaer & Harten & John A. Waterston & Yvonne W. Wu & $\mathrm{MD}$ \\
\hline
\end{tabular}


Jeff Burns, MD, MS

Ted Burns, MD

Jason Crowell, MD, MPA

Gregg Day, MD

Alberto Espay, MD, MSc
Eoin Flanagan, MB $\mathrm{BCh}$

Katherine Fu, MD

Eric Goldstein, MD

Katrina Ignacio, MD

Vikram Karnik, MD

\section{Pearce Korb, MD, MHPE, FAAN \\ Erica Marini, PharmD, MS, BCPS}

Alex Menze,

$\mathrm{MD}$
Tesha Monteith, MD, FAHS,

FAAN

Fábio Nascimento, $\mathrm{MD}$

M. Will Rondeau, PA

Meagan Seay, DO
Gordon Smith, MD, FAAN

Andrew Southerland, $\mathrm{MD}$

Derek Stitt, MD

Jon Stone, MB, ChB, FRCP, PhD

\section{Disclosures}

Full disclosures are available at Neurology.org/N. 


\title{
Neurology
}

\author{
Message From the Editors to Our Reviewers \\ José G. Merino, Olga Ciccarelli, Bradford B. Worrall, et al. \\ Neurology 2022;98;3-11 \\ DOI 10.1212/WNL.0000000000013044
}

This information is current as of December 27, 2021

$\begin{array}{ll}\begin{array}{l}\text { Updated Information \& } \\ \text { Services }\end{array} & \begin{array}{l}\text { including high resolution figures, can be found at: } \\ \text { http://n.neurology.org/content/98/1/3.full }\end{array} \\ \text { Permissions \& Licensing } & \begin{array}{l}\text { Information about reproducing this article in parts (figures,tables) or in } \\ \text { its entirety can be found online at: } \\ \text { http://www.neurology.org/about/about_the_journal\#permissions }\end{array} \\ & \begin{array}{l}\text { Information about ordering reprints can be found online: } \\ \text { http://n.neurology.org/subscribers/advertise }\end{array}\end{array}$

Neurology ${ }^{\circledR}$ is the official journal of the American Academy of Neurology. Published continuously since 1951, it is now a weekly with 48 issues per year. Copyright @ 2021 American Academy of Neurology. All rights reserved. Print ISSN: 0028-3878. Online ISSN: 1526-632X.

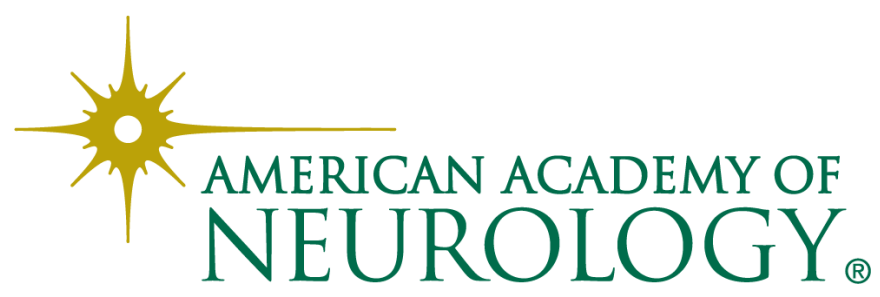

